Communications in Physics, Vol. 24, No. 1 (2014), pp. 1-8

\title{
THE BULK METRIC IN THE THEORY WITH TWO EXTRA DIMENSIONS
}

\author{
TRAN HUU PHAT \\ Vietnam Atomic Energy Institute, 59 Ly Thuong Kiet, Hanoi, Vietnam \\ PHAN HONG LIEN \\ Military Academy of Technology, 236 Hoang Quoc Viet, Hanoi, Vietnam \\ HA VAN CHUNG \\ Hanoi National University of Education, 136 Xuan thuy, Hanoi, Vietnam \\ E-mail: tranhuuphat41@gmail.com
}

Received 25 December 2013

Accepted for publication 22 February 2014

\begin{abstract}
The metric of a warped space-time with two extra dimensions is established by means of the Einstein equations in six dimensions and the compactification of two extra dimensions on a square. It is shown that at every among two fixed points our manifold reduces to the five-dimensional Randall - Sundrum space-time and the hierarchy problem could be solved.
\end{abstract}

Keywords: six dimensions, 6D Riemann manifold, bulk metric, compactification.

\section{INTRODUCTION}

The first idea of extra dimensions, as it is well known, have been proposed by Kaluza Klein in the 1920s to unify electromagnetism and gravity [1]. The expansion of space-time dimensions beyond the four is one of most interesting fields of modern theoretical physics.

Besides the extended models of Standard Model (SM), the extra dimensions have been considered as the ways to investigate both old and open problems of particle physics and Cosmology, in the new physics beyond the SM to unify gravity with gauge interactions. Extra dimensions provide the solutions of hierarchy problem in the 5D Randall-Sundrum space-time [2], or in more extra dimensions [3]; the explanation of dark matter [4,5], neutrino physics in the 6D Universal Extra Dimensions (UED) model [6-9] ; the expective proposal for GUT based on String Theories with $10 \mathrm{D}$ or 11D [10,12]. Extra dimensional theories can lead to really significant physical consequences.

Starting from two following important observations

1) Solving the mass hierarchy problem by means of the wrap factor attaching to the four dimensional metric is very successful $[2,13]$;

2) The proton stability is explained within the framework of a theory with two extra dimensions [14,15],

(C)2014 Vietnam Academy of Science and Technology 
we assume that the geometry of our theory is a 6D Riemann manifold, whose metric is expressed as

$$
d s^{2}=e^{-2 f\left(x_{5}, x_{6}\right)} g_{\mu \nu} d x^{\mu} d x^{\nu}-d x_{5}^{2}-d x_{6}^{2}
$$

We focus on configurations where symmetry in the brane direction is maximum

$$
\begin{aligned}
d s^{2} & =G_{A B} d x^{A} d x^{B}=e^{-2 f} g_{\mu \nu} d x^{\mu} d x^{\nu}+g_{m n} d x^{m} d x^{n} \\
& =e^{-2 f} g_{\mu \nu} d x^{\mu} d x^{\nu}+e^{2 p} d z d \bar{z} .
\end{aligned}
$$

where $g_{\mu \nu}=\operatorname{diag}(1,-1,-1,-1)$ is the 4- dimensional tensor metric. The coordinates are $x^{A}=$ $x^{\mu}, x^{5}, x^{6}$ with $\mu, \nu=0,1,2,3$ labelling the brane directions, and $m, n=5,6$ (or $z=x^{5}+i x^{6}$ ) being coordinates for the two dimensions transverse to the branes.

$$
\begin{aligned}
d s^{2}= & G_{A B} d x^{A} d x^{B} \\
= & G_{\mu \nu} d x^{\mu} d x^{\nu}+\left(G_{\mu 5}+G_{5 \mu}\right) d x^{\mu} d x^{5}+\left(G_{\mu 6}+G_{6 \mu}\right) d x^{\mu} d x^{6} \\
& +G_{56} d x^{5} d x^{6}+G_{65} d x^{6} d x^{5}+G_{55} d x^{5} d x^{5}+G_{66} d x^{6} d x^{6}
\end{aligned}
$$

where

$$
G_{\mu \nu}=e^{-2 f\left(x_{5}, x_{6}\right)} g_{\mu \nu} ; G_{55}=-1 ; G_{66}=-1 ; G_{56}=G_{65}=0 ;
$$

The six dimensional metric reads

$$
d s^{2}=e^{-2 f\left(x_{5}, x_{6}\right)} g_{\mu \nu} d x^{\mu} d x^{\nu}-d x_{5}^{2}-d x_{6}^{2},
$$

where $0 \leq x_{5}, x_{6} \leq L$.

To extend the field equation in 6 - dimensional space-time, we start from the Einstein's equation

$$
R_{\mu \nu}-\frac{1}{2} R g_{\mu \nu}+\Lambda g_{\mu \nu}=8 \pi G T_{\mu \nu}
$$

The general 6 - dimensional action takes the form

$$
S=S_{\text {grav }}+S_{\text {hid }}+S_{v i s},
$$

where

$$
\begin{aligned}
S_{\text {grav }} & =\int d^{4} x \int d x_{5} d x_{6} \sqrt{-G}\left[-\Lambda+2 M^{4} R\right] \\
S_{\text {hid }} & =\int d^{4} x \int d x_{5} d x_{6} \sqrt{-g_{\text {hid }}}\left[L_{h i d}-V_{\text {hid }}\right] \\
S_{\text {vis }} & =\int d^{4} x \int d x_{5} d x_{6} \sqrt{-g_{v i s}}\left[L_{v i s}-V_{v i s}\right]
\end{aligned}
$$

In the classical case, the particle excitation is absent, ie. $L_{\text {hid }}=L_{v i s}=0$

$$
\begin{aligned}
S= & \int d^{4} x \int d x_{5} d x_{6} \sqrt{-G}\left[\left(-\Lambda+2 M^{4} R\right)\right. \\
& \left.-\sqrt{-g_{v i s}} V_{v i s} \delta\left(x_{5}-L\right) \delta\left(x_{6}-L\right)-\sqrt{-g_{h i d}} V_{h i d} \delta\left(x_{5}\right) \delta\left(x_{6}\right)\right]
\end{aligned}
$$


The 6 - dimensional Einstein's Equation is derived from $\delta S=0$.

$$
\begin{aligned}
\sqrt{-G}\left(R_{A B}-\frac{1}{2} G_{A B} R\right)= & \frac{1}{4 M^{4}}\left[\Lambda \sqrt{-G} G_{A B}\right. \\
& +V_{v i s} \sqrt{-g_{v i s}} g_{\mu \nu}^{v i s} \delta_{A}^{\mu} \delta_{B}^{\nu} \delta\left(x_{5}-r_{c}\right) \delta\left(x_{6}\right) \\
& \left.+V_{\text {hid }} \sqrt{-g_{\text {hid }}} g_{\mu \nu}^{\text {hid }} \delta_{A}^{\mu} \delta_{B}^{\nu} \delta\left(x_{5}\right) \delta\left(x_{6}\right)\right]
\end{aligned}
$$

where the $(3+1)$ - dimensional space-time is connected with the $(4+2)$ - dimensional by

$$
\begin{aligned}
& g_{\mu \nu}^{v i s}(x)=G_{A B}\left(x, x_{5}=L, x_{6}=L\right), \\
& g_{\mu \nu}^{\text {hid }}(x)=G_{A B}\left(x, x_{5}=0, x_{6}=0\right) .
\end{aligned}
$$

Our aim is to determine the analytical expression for $f\left(x_{5}, x_{6}\right)$ so that above metric is a solution of Einstein's equation extended in six dimensions. It is shown that this metric could be reduced to the RS space-time in the cases of compactifications on two dimensional sphere $\left(S^{2} / Z_{2} 2\right)$ or on a square $\left(T^{2} / Z_{2}\right)$. We also obtained the physical consequences when the $6 \mathrm{D}$ metric is considered by the 4D effective theory. Similarly in the case of RS space-time, the hierarchy problem is solved due to the Planck mass.

This paper is organized as follows. In Sec. II the Einstein equations extended in 6D spacetime are derived from the 6D Riemann manifold. These equations with two extra dimension have been solved in the cylindrical coordinate system. Sec. III is devoted to investigate the hierarchy problems. The discussions and conclusions are given in Sec. IV.

\section{THE SIX - DIMENSIONAL SOLUTION OF EINSTEIN'S EQUATION IN THE CYLINDRICAL SYMMETRY}

We impose two external variables in the cylindrical coordinates system

$$
x^{5}=\rho \cos \theta ; x^{6}=\rho \sin \theta ;
$$

where $0 \leq \rho \leq r_{c},-\pi \leq \theta \leq \pi, f\left(x_{5}, x_{6}\right)=f(\rho, \theta)$.

This leads to the following metric

$$
d s^{2}=e^{-2 f(\rho, \theta)} g_{\mu \nu} d x^{\mu} d x^{\nu}-d \rho^{2}-\rho^{2} d \theta^{2} .
$$

We determine the bulk metric which is the solution of 6 - dimensional Einstein's equation

$$
\begin{aligned}
\sqrt{-G} & \left(R_{A B}-\frac{1}{2} G_{A B} R\right)=\frac{1}{4 M^{4}}\left[\Lambda \sqrt{-G} G_{A B}\right. \\
& \left.+V_{v i s} \sqrt{-g_{v i s}} g_{\mu \nu}^{v i s} \delta_{A}^{\mu} \delta_{B}^{\nu} \delta\left(\rho-r_{c}\right) \delta(\theta-\pi)+V_{h i d} \sqrt{-g_{h i d}} g_{\mu \nu}^{h i d} \delta_{A}^{\mu} \delta_{B}^{\nu} \delta(\rho) \delta(\theta)\right],
\end{aligned}
$$

where

$$
\begin{aligned}
& g_{\mu \nu}^{v i s}\left(x^{\mu}\right)=G_{\mu \nu}\left(x^{\mu}, \rho=r_{c}, \theta=\pi\right), \\
& g_{\mu \nu}^{\text {hid }}\left(x^{\mu}\right)=G_{\mu \nu}\left(x^{\mu}, \rho=0, \theta=0\right),
\end{aligned}
$$




$$
\begin{aligned}
d s^{2}= & G_{A B} d x^{A} d x^{B} \\
= & G_{\mu \nu} d x^{\mu} d x^{\nu}+2 G_{\mu \rho} d x^{\mu} d x^{\rho}+2 G_{\mu \theta} d x^{\mu} d x^{\theta} \\
& \left(G_{\rho \theta}+G_{\theta \rho}\right) \rho d \rho d \theta+G_{\rho \rho} d \rho^{2}+G_{\theta \theta} d \theta^{2} .
\end{aligned}
$$

Here $G_{\rho \theta}=G_{\theta \rho}=0, G_{\mu \rho}=G_{\mu \theta}=0$ in zero mode.

$$
d s^{2}=G_{\mu \nu} d x^{\mu} d x^{\nu}+G_{\rho \rho} d \rho^{2}+G_{\theta \theta} d \theta^{2},
$$

where

$$
G_{\mu \nu}=g_{\mu \nu} e^{-2 f(\rho, \theta)} ; G_{\rho \rho} d \rho^{2}=-d \rho^{2} ; G_{\rho \rho}=-1 ; G_{\theta \theta}=-r_{c}^{2} d \theta^{2} ; G_{\theta \theta}=-r_{c}^{2} .
$$

The six - dimensional metric reads

$$
d s^{2}=g_{\mu \nu} e^{-2 f(\rho, \theta)} d x^{\mu} d x^{\nu}-d \rho^{2}-r_{c}^{2} d \theta^{2} .
$$

Hence

$$
\begin{aligned}
& G_{A B}=\operatorname{diag}\left(e^{-2 f(\rho, \theta)},-e^{-2 f(\rho, \theta)},-e^{-2 f(\rho, \theta)},-e^{-2 f(\rho, \theta)},-1,-r_{c}^{2}\right), \\
& \begin{aligned}
G^{A B}=\operatorname{diag}\left(e^{2 f(\rho, \theta)},-e^{2 f(\rho, \theta)},-e^{2 f(\rho, \theta)},-e^{2 f(\rho, \theta)},-1,-r_{c}^{2}\right), \\
g_{\mu \nu}^{v i s}\left(x^{\mu}\right)=G_{A B}\left(x^{\mu}, \rho=r_{c}, \theta=\pi\right) \\
=G_{\mu \nu}\left(\rho=r_{c}, \theta=\pi\right)=g_{\mu \nu} e^{-2 f\left(r_{c}, \pi\right)} \\
g_{\mu \nu}^{h i d}\left(x^{\mu}\right)=G_{A B}\left(x^{\mu}, \rho=0, \theta=0\right) \\
=G_{\mu \nu}(\rho=0, \theta=0)=g_{\mu \nu} e^{-2 f(0,0)} \\
G=\operatorname{det} G_{A B}=-r_{c}^{2} e^{-8 f(\rho, \theta)} \Rightarrow \sqrt{-G}=r_{c} e^{-4 f(\rho, \theta)} .
\end{aligned}
\end{aligned}
$$

The affine connections (or Christoffel notation) in 6 - dimensional space-time are defined by

$$
\begin{aligned}
\Gamma_{B C}^{A} & =\frac{1}{2} G_{A D}\left(\partial_{B} G_{C D}+\partial_{C} G_{D B}-\partial_{D} G_{B C}\right), \\
\Gamma_{B C}^{A} & =G_{A D} \Gamma_{D, B C}
\end{aligned}
$$

The nonvanishing Christoffel symbols are (in the linear approximation)

$$
\begin{aligned}
& \Gamma_{05}^{0}=\Gamma_{50}^{0}=\Gamma_{15}^{1}=\Gamma_{51}^{1}=\Gamma_{25}^{2}=\Gamma_{52}^{2}=\Gamma_{35}^{3}=\Gamma_{53}^{3}=-f_{\rho}^{\prime}(\rho, \theta), \\
& \Gamma_{06}^{0}=\Gamma_{60}^{0}=\Gamma_{16}^{1}=\Gamma_{61}^{1}=\Gamma_{26}^{2}=\Gamma_{62}^{2}=\Gamma_{36}^{3}=\Gamma_{63}^{3}=-f_{\rho}^{\prime}(\rho, \theta), \\
& \Gamma_{00}^{5}=-f_{\rho}^{\prime}(\rho, \theta) e^{-2 f(\rho, \theta)}, \\
& \Gamma_{11}^{5}=\Gamma_{22}^{5}=\Gamma_{33}^{5}=-f_{\rho}^{\prime}(\rho, \theta) e^{-2 f(\rho, \theta)}, \\
& \Gamma_{00}^{6}=-\frac{1}{r_{c}^{2}} f_{\theta}^{\prime}(\rho, \theta) e^{-2 f(\rho, \theta)}, \\
& \Gamma_{11}^{6}=\Gamma_{22}^{6}=\Gamma_{33}^{6}=\frac{1}{r_{c}^{2}} f_{\rho}^{\prime}(\rho, \theta) e^{-2 f(\rho, \theta)} .
\end{aligned}
$$

The components of the Ricci tensor are defined by

$$
R_{A B}=R_{A B C}^{C}=\partial_{B} \Gamma_{A C}^{C}+\Gamma_{A C}^{D} \Gamma_{B D}^{C}-\Gamma_{A B}^{C} \Gamma_{C D}^{D} .
$$


The elements of the Ricci tensor are

$$
\begin{aligned}
R_{\mu \nu}= & g_{\mu \nu} e^{-2 f(\rho, \theta)}\left[f{ }^{\prime}(\rho, \theta)+\frac{1}{r_{c}^{2}} f^{\prime}{ }_{\theta}(\rho, \theta)-4 f_{\rho}^{\prime 2}(\rho, \theta)-\frac{1}{r_{c}^{2}} f_{\theta}^{\prime 2}(\rho, \theta)\right], \\
R_{\rho \rho}= & 4\left[-f{ }_{\rho}(\rho, \theta)+f_{\rho}^{\prime 2}(\rho, \theta)\right] \\
R_{\theta \theta}= & 4\left[-f{ }_{\rho}(\rho, \theta)+f_{\theta}^{\prime 2}(\rho, \theta)\right] \\
R_{\mu \rho}= & R_{\rho \mu}=R_{\mu \theta}=R_{\theta \mu}=R_{\rho \theta}=R_{\theta \rho}=0 \\
& R=G^{A B} R_{A B}=G^{\mu \nu} R_{\mu \nu}+G^{\rho \rho} R_{\rho \rho}+G^{\theta \theta} R_{\theta \theta} \\
& R=4\left[2 f{ }_{\rho}(\rho, \theta)+\frac{2}{r_{c}^{2}} f^{\prime}{ }_{\theta}(\rho, \theta)-5 f_{\rho}^{\prime 2}(\rho, \theta)-\frac{5}{r_{c}^{2}} f_{\theta}^{\prime 2}(\rho, \theta)\right]
\end{aligned}
$$

Substituting (23) - (31) into the 6D Einstein's equations (16), (17), we have

$$
\begin{aligned}
& r_{c} e^{-4 f \rho, \theta}\left[\left(\begin{array}{ccc}
R_{\mu \nu} & 0 & 0 \\
0 & R_{\rho \rho} & 0 \\
0 & 0 & R_{\theta \theta}
\end{array}\right)-\frac{1}{2}\left(\begin{array}{ccc}
G_{\mu \nu} & 0 & 0 \\
0 & G_{\rho \rho} & 0 \\
0 & 0 & G_{\theta \theta}
\end{array}\right) R\right] \\
= & -\frac{1}{4 M^{4}}\left[\Lambda r_{c} e^{-4 f \rho, \theta}\left(\begin{array}{ccc}
G_{\mu \nu} & 0 & 0 \\
0 & G_{\rho \rho} & 0 \\
0 & 0 & G_{\theta \theta}
\end{array}\right)\right. \\
& +V_{v i s} e^{-4 f\left(r_{c}, \pi\right)} g_{\mu \nu} e^{-2 f\left(r_{c}, \pi\right)} \delta_{A}^{\mu} \delta_{B}^{\nu} \delta\left(\rho-r_{c}\right) \delta(\theta-\pi) \\
& \left.+V_{\text {hid }} e^{-4 f(0,0)} g_{\mu \nu} e^{-2 f(0,0)} \delta_{A}^{\mu} \delta_{B}^{\nu} \delta(\rho) \delta(\theta)\right],
\end{aligned}
$$

By separating the indices $\theta \theta$ and $\rho \rho$, we obtain the system of equations

$$
\begin{aligned}
4 f^{\prime \prime}{ }_{\rho}(\rho, \theta)+10 f_{\rho}^{\prime 2}(\rho, \theta)+\frac{6}{r_{c}^{2}} f_{\theta}^{\prime 2}(\rho, \theta) & =-\frac{\Lambda}{4 M^{4}} . \\
-\frac{4}{r_{c}^{2}} f^{\prime \prime}{ }_{\theta}(\rho, \theta)+\frac{10}{r_{c}^{2}} f_{\theta}^{\prime 2}(\rho, \theta)+6 f_{\rho}^{\prime 2}(\rho, \theta) & =-\frac{\Lambda}{4 M^{4}} .
\end{aligned}
$$

Similarly for indices $\mu \nu$, we have

$$
\begin{aligned}
& 3 f{ }_{\rho}(\rho, \theta)+\frac{3}{r_{c}^{2}} f{ }_{\theta}(\rho, \theta)-6 f_{\rho}^{\prime 2}(\rho, \theta)-\frac{6}{r_{c}^{2}} f_{\theta}^{\prime 2}(\rho, \theta) \\
= & -\frac{\Lambda}{4 M^{4}}+\frac{V_{v i s}}{4 M^{4} r_{c}} \delta\left(\rho-r_{c}\right) \delta(\theta-\pi)+\frac{V_{h i d}}{4 M^{4} r_{c}} \delta(\rho) \delta(\theta) .
\end{aligned}
$$

It is clearly that when $\rho$ coordinate is absent, i.e, the sixth dimension disappears, equations (30) - (32) reduce to the Einstein's equation in the RS space-time.

Note that the isotropy in the $\left(x_{5}, x_{6}\right)$ - plane requires that $f$ does not depend on the angle $\theta$ in the pole coordinate system $x_{5}=\rho \cos \theta, x_{6}=\rho \sin \theta$. In other words, $f$ must be a function of the proper distance $\rho$, i.e.

and it satisfies the conditions

$$
f=f(\rho)
$$

$$
f\left(\rho-r_{c}\right)=f\left(r_{c}-\rho\right), \quad \delta(\theta)=\delta(\theta \pm \pi) .
$$


Eq. (34) becomes

$$
6 f_{\rho}^{\prime 2}(\rho)=-\frac{\Lambda}{4 M^{4}}
$$

Its solution is

$$
f(\rho)=k \rho .
$$

Here the single scale $k$ is related to cosmological constant $\Lambda$ by

$$
k^{2}=-\frac{\Lambda}{24 M^{4}},
$$

or, equivalently $\Lambda=-24 k^{2} M^{4}$.

We consider $f(\rho)$ in the interval $\left(0, r_{c}\right)$, it is easily to see that

$$
f^{\prime \prime}(\rho)=-\frac{2 k}{r_{c}}\left[\delta\left(\rho-r_{c}\right)-\delta(\rho)\right]
$$

Substituting (39) into (35), we obtain

$$
V_{v i s}=-24 M^{4} k ; \quad V_{\text {hid }}=24 M^{4} k .
$$

The solution for the bulk metric reads

$$
d s^{2}=e^{-2 k \rho} g_{\mu \nu} d x^{\mu} d x^{\nu}-d \rho^{2}-\rho^{2} d \theta^{2} .
$$

Generally, the solution - metric of Einstein's equation in 6 dimensional space-time, takes the form

$$
d s^{2}=e^{-2 k|z|} g_{\mu \nu} d x^{\mu} d x^{\nu}-d x_{5}^{2}-d x_{6}^{2} .
$$

or $d s^{2}=e^{-2 k \sqrt{x_{5}^{2}+x_{6}^{2}}} g_{\mu \nu} d x^{\mu} d x^{\nu}-d x_{5}^{2}-d x_{6}^{2}$.

It is clearly that (42) will reduce to the RS metric in the following cases:

1 , Compactification on a 2 - dimensional sphere $\left(S^{2} / Z_{2}\right)$

$$
x_{5}^{2}+x_{6}^{2} \leq r_{c}^{2} .
$$

At $x_{6}=0$ we have $-r_{c} \leq x_{5} \leq r_{c}$, then the metric (42) will takes the form

$$
d s^{2}=e^{-2 k|\phi|} g_{\mu \nu} d x^{\mu} d x^{\nu}-r_{c}^{2} d \phi^{2},
$$

in which $x_{5}=r_{c} \phi,-1 \leq \phi \leq 1$.

Metric (43) is exactly the RS metric if we identify (43) at $\theta=1$ with that at $\theta=-1$. Hence, the manifold (42) contains two RS space-time (43) as its submanifolds at $x_{5}=0$ and $x_{6}=0$.

2 , Compactification on a square $\left(T^{2} / Z_{2}\right): 0 \leq x_{5}, x_{6} \leq L$.

In this case (42) is also to be reduced to (43) at $x_{5}=0, L$ and $x_{6}=0, L$. As a result, the manifold (42) contains two RS manifolds at the square summits if we identify the point $x_{5}=0$ with $x_{5}=L$ and the point at $x_{6}=0$ with $x_{6}=L$. 


\section{THE PLANCK MASS AND HIERARCHY PROBLEM}

We consider scalar field $T(x)$ so that its VEV is the compact radius $r_{c}=$ const. The local 4 - dimensional metric is given by

$$
g_{\mu \nu}=\eta_{\mu \nu}+h_{\mu \nu}
$$

where $h_{\mu \nu}$ is symmetric tensor and very small. It is identified to physical gravitational field $h_{\mu \nu}$ of 4 - dimensional effective theory, $\left|h_{\mu \nu}\right| \ll 1$.

The 6 - dimensional metric takes the form

$$
d s^{2}=e^{-2 k|T(x)|}\left[\eta_{\mu \nu}+h_{\mu \nu}\right] d x^{\mu} d x^{\nu}-d T^{2}(x)-T^{2}(x) d \theta^{2} .
$$

Note that this metric is locally the same as the "vacuum" solution.

By substituting metric (45) into the original action (7), the four dimensional effective theory as follows

$$
S_{\text {grav }}=\int d^{4} x \int d T(x) \int d \theta \sqrt{-G}\left[-\Lambda+2 M^{4} R\right],
$$

where

$$
\begin{aligned}
\sqrt{-G} & =T(x) e^{-4 k|T(x)|} \sqrt{-\bar{g}}, \bar{g}=\operatorname{det}_{\mu \nu}, \\
R & =e^{2 k|T(x)|} \bar{R}, G_{\mu \nu}=e^{-2 k|T(x)|} g_{\mu \nu} .
\end{aligned}
$$

Here $\bar{R}$ is 4 - dimensional Ricci scalar made out of $g_{\mu \nu}$, in contrast to the six - dimensional Ricci scalar $R$, made out of $G_{A B}(x, T)$.

Substituting (47) into (46) the effective action is given by

$$
S_{\text {eff }}=\int d^{4} x \int d T(x) \int r_{c} d \theta \sqrt{-\bar{g}} e^{-4 k|T(x)|}\left[-\Lambda+2 M^{4} e^{2 k|T(x)|} \bar{R}\right] .
$$

We focus on the mass term in the 4 - dimensional gravitational action

$$
S_{\text {grav }}=-\int d^{4} x \sqrt{-\bar{g}} 2 M_{P l}^{2} \bar{R}
$$

where $M_{P l}$ is 4 - dimensional Planck mass.

Compare (48) to (49), we derive

$$
M_{P l}^{2}=-M^{4} \int_{-\pi}^{\pi} r_{c} d \theta \int e^{-2 k|T(x)|} d T(x)=\frac{M^{4} r_{c}}{2 k} \int_{-\pi}^{\pi} d \theta e^{-2 k|T(x)|},
$$

With $|T(x)|=r_{c}|\theta|$, it becomes

$$
M_{P l}^{2}=\left.\frac{M^{4} r_{c}}{2 k}\left(-\frac{1}{2 k r_{c}}\right) 2 e^{-2 k r_{c} \theta}\right|_{0} ^{\pi}=\frac{M^{4}}{2 k^{2}}\left(1-e^{-2 k r_{c} \pi}\right) .
$$

Similarly in the case of 5 dimensions the result (51) shows that $M_{P l}$ depends only weakly on $r_{c}$ in the large $k r_{c}$ limit. By means an adequate value of $k$ the 6 - dimensional mass $M^{4}$ has the same power with 4 - dimensional Planck mass. That means the hierarchy problem could be solved. 


\section{DISCUSSION AND CONCLUSION}

In this paper we built a formalism for the warped six-dimensional space-time with a square of compactification. In addition to the hierarchy problem that could be solved successfully, the formalism still encompasses many other features:

1. It allows the existence of chiral fermions [14] in the four - dimensional effective theory. This property has good prospect for setting up the six-dimensional standard model within a warped space-time.

2. The implication of the compactification on a square implies that for standard model in six dimensions the symmetry ensures a lifetime of proton longer than the current experimental bounds [15], even in the presence of baryon violation at $\mathrm{TeV}$ scale and forces the neutrino masses to be of the Dirac type [9]. It also explains the origin of electroweak symmetry breaking [16], the number of fermion generation [17] and the breaking of grand unified gauge groups [18].

Hence, it is hopeful that our proposed theory could pave the way for better understanding various physical phenomena of high energy physics.

\section{REFERENCES}

[1] Th. Kaluza, Sitzungober. Preuss. Akad. Wiss. Berlin. (1921) 966; O. Klein, Z. Phys. 37 (1926) 895.

[2] L. Randall and R. Sundrum, Phys. Rev. Lett. 83 (1999) 3370.

[3] H. Hatanaka, T. Inami, and C. S. Lim, Mod. Phys. Lett. A 13 (1998) 2601 - 2612.

[4] N. Arkani - Hamed, S. Dimopoulos, and G. R. Dvali, Phys. Lett. B 429 (1998) 263.

[5] T. Appelquist and H. C. Cheng and B. A. Dobrescu, Phys. Rev. D 64 (2001), 035002.

[6] S. C. Park and J. Shu, Phys. Rev. D 79 (2009) 091702, 0901.0702.

[7] J. Appelquist, H. U.Yee, Phys. Rev. D 67 (2003) 055002.

[8] G. Cacciapaglia, A. Deandrea and J. Llodra-Perez, JHEP 1003 (2010) 083 [arXiv:0907.4993 [hep-ph]]

[9] T. Appelquist, B. A. Dobrescu, E. Ponton, and H. U. Yee, Phys. Rev. D 65 (2002) 105019.

[10] A. Hebecker, JHEP 01 (2004) 047.

[11] A. Pomarol and M. Quiros, Phys. Lett. B 438 (1998) 225.

[12] A. Bayntun, C. P. Burgess and L. Var Nierop, New J. Phys. 12 (2010) 075015.

[13] K. Choi, I-W. Kim, and S. Chang, New J. Phys. 1292010) 075014.

[14] B. A. Dobrescu and E. Ponton, (2004).

[15] T. Appelquist, B. A. Dobrescu, E. Ponton, H. U. Lee, Phys. Rev. Lett. (2001) 181802.

[16] N. Arkani-Hamed , H. C. Cheng, B. A. Dobrescu and L. J. Hall, Phys. Rev. D 62, 096006 (2000).

[17] B. Dobrescu and E. Poppitz, Phys. Rev. Lett. 87 (2001) 031801.

[18] T. Asaka, W. Buchmuller, and L. Covi, Nucl. Phys. B 648 (2003) 231. 